\title{
Human equilibrative nucleoside transporter 1 gene expression is associated with gemcitabine efficacy in advanced leiomyosarcoma and angiosarcoma
}

Bruno Vincenzi ${ }^{*}, 1$, Silvia Stacchiotti ${ }^{2}$, Paola Collini ${ }^{3}$, Francesco Pantano ${ }^{1}$, Carla Rabitti ${ }^{4}$, Giuseppe Perrone ${ }^{4}$, Michele Iuliani ${ }^{5}$, Alfonso Baldi ${ }^{5}$, Giuseppe Badalamenti ${ }^{6}$, Roberta Sanfilippo ${ }^{2}$, Daniele Santini ${ }^{1}$, Andrea Onetti Muda ${ }^{4}$, Alessandro Gronchi ${ }^{7}$, Paolo Casali ${ }^{2}$, Angelo Paolo Dei Tos ${ }^{8}$ and Giuseppe Tonini ${ }^{1}$

${ }^{1}$ Medical Oncology, Campus Bio-Medico University of Rome, Via Alvaro del Portillo 200, 00128 Rome, Italy; ${ }^{2}$ Sarcoma Unit Cancer Medicine Department, Fondazione IRCCS Istituto Nazionale Tumori, Via Venezian 1, 20133 Milan, Italy; ${ }^{3}$ Department of Diagnostic Pathology and Laboratory Medicine, Fondazione IRCCS Istituto Nazionale dei Tumori, Via Venezian 1, 20133 Milan, Italy; ${ }^{4}$ Human Pathology, Campus Bio-Medico University of Rome, Via Alvaro del Portillo 200, 00128 Rome, Italy; ${ }^{5}$ Department of Environmental, Biological and Pharmaceutical Sciences and Technologies, Second University of Naples, Via A. Vivaldi 43, 81100 Caserta, Italy; ${ }^{6}$ Department of Surgical and Oncological Sciences, University of Palermo, Via del Vespro 131, 90127 Palermo, Italy; ${ }^{7}$ Department of Surgery, Fondazione IRCCS Istituto Nazionale dei Tumori, Via Venezian 1, 20133 Milan, Italy and ${ }^{8}$ Department of Pathology and Molecular Genetics, Treviso General Hospital, Piazza Ospedale 1, 31100 Treviso, Italy

Background: The expression of human equilibrative nucleoside transporter 1 (hENT1), the major gemcitabine transporter into cells, has been thoroughly investigated as a predictive marker of response to gemcitabine in pancreatic cancer and biliary tract cancers. Since gemcitabine is widely used in the treatment of leiomyosarcoma and angiosarcoma, we investigated the correlation between hENT1 expression and gemcitabine efficacy in these sarcoma subtypes.

Methods: We retrospectively identified 71 patients affected by advanced angiosarcoma (26) or leiomyosarcoma (45) treated within five Italian referral centres for sarcoma; among them, 49 patients (15 angiosarcoma, 34 leiomyosarcoma) were treated with gemcitabine. All tumour samples were analysed for $h E N T 1$ expression by real-time PCR. Median $2^{-\Delta C t}$ value was used as the cutoff to dichotomise patients into 'high' expression and 'low' expression groups. Kaplan-Meier analysis was performed to estimate progression-free survival (PFS) and overall survival (OS).

Results: We found a significant association between high hENT1 expression levels and favourable outcome in terms of PFS and OS compared to cases with low hENT1 expression in leiomyosarcoma treated with gemcitabine (PFS: 6.8 vs 3.2 months, $P=0.004$; OS: 14.9 vs 8.5 months, $P=0.007)$. In addition, hENT1 overexpression correlated with a significant improvement in PFS (9.3 vs 4.5 months; $P=0.02)$ and $O S(20.6$ vs 10.8 months; $P=0.001)$ in angiosarcoma patients treated with gemcitabine.

Conclusions: Our study suggests that higher hENT1 expression are associated to gemcitabine efficacy both in patients with advanced leiomyosarcoma and angiosarcoma.

*Correspondence: Professor B Vincenzi; E-mail: b.vincenzi@unicampus.it

Received 27 March 2017; revised 23 May 2017; accepted 31 May 2017; published online 22 June 2017

(C) 2017 Cancer Research UK. All rights reserved 0007-0920/17 
The human equilibrative nucleoside transporter 1 (hENT1) is a transmembrane protein that is thought to be responsible for the intracellular uptake of the prodrug gemcitabine into tumour cells (Ueno et al, 2007). Other identified transporters belong to the human concentrative nucleoside transporter family (hCNTs), especially hCNT1 (Bhutia et al, 2011) but hENT1 is generally considered to be predominantly involved in gemcitabine transport. The first study exploring the relationship between hENT1 and gemcitabine efficacy was published in 2004 (Spratlin et al, 2004) and, during the following decade, several pre-clinical studies and clinical investigations confirmed the hypothesis that hENT1 overexpression might serve as a predictive biomarker for the efficacy of gemcitabine (Michalski et al, 2008; Pérez-Torras et al, 2008; Farrell et al, 2009; Maréchal et al, 2009, 2012; Morinaga et al, 2012; Nakagawa et al, 2013). In particular in a series of 105 patients with pancreatic adenocarcinoma treated with gemcitabine, the high tumour expression of hENT1 was associated with a significant improvement in overall survival (OS) (25.7 vs 8.4 months), progression-free survival (PFS) (12.6 vs 5.8 months) and DiseaseFree Survival (20.4 vs 9.2 months; Giovannetti et al, 2006). Similar results have been obtained for biliary tract cancers in a retrospective analysis of 44 patients with locally advanced or metastatic cholangiocarcinoma undergoing treatment with gemcitabine, where higher hENT1 positivity was correlated with a better PFS (6.0 vs 2.0 months) and OS (11.0 vs 5.0 months; Borbath et al, 2012).

Our group has previously demonstrated that patients affected by locally advanced biliary tract cancers, who were hENT1 positive, showed significant benefits in terms of PFS (6.33 vs 2.83 months) (Santini et al, 2011). Moreover, we identified hENT1 as a negative prognostic marker in patients with ampullary and gastric cancer post radical resection and as a potential predictive factor to select patients who could benefit from chemotherapy postoperatively (Santini et al, 2008, 2010; Perrone et al, 2010.

During the last few years, some investigators reported that hENT1 might potentially act as a prognostic rather than as a predictive marker. Indeed, a significant association between hENT1 expression and clinical outcome was demonstrated in patients with resected pancreatic cancer (Kim et al, 2011) and in chemotherapy-naive gallbladder adenocarcinoma patients (Espinoza et al, 2016); on the contrary no evidence for hENT1 as a prognostic biomarker was found in pancreatic cancer patients treated with gemcitabine-based neoadjuvant chemoradiotherapy (Kawada et al, 2012).

Up to now, translational hENT1 data have been reported from three controlled clinical trials in pancreatic cancer. Two retrospective studies conducted in the adjuvant setting, demonstrated a significant correlation between hENT1 and benefit from gemcitabine treatment after surgical resection (Farrell et al, 2009; Greenhalf et al, 2014), whereas the third study did not confirm its predictive value in a prospective series of metastatic patients treated with gemcitabine (Poplin et al, 2013).

hENT1 as prognostic/predictive biomarker has been also investigated in patients affected by non-small cell lung cancer and metastatic bladder cancer and its expression was significantly associated with gemcitabine treatment efficacy (Oguri et al, 2007; Matsumura et al, 2011).

No data concerning hENT1 expression in soft tissue sarcomas (STSs) are available, although gemcitabine is an active drug in different sarcoma subtypes, including leiomyosarcoma (Pautier et al, 2012) and angiosarcoma (Hensley et al, 2002, 2008; Stacchiotti et al, 2012).

The aim of this study is to retrospectively determine the gene expression levels of hENT1 in samples derived from advanced leiomyosarcoma and angiosarcoma patients and, for those treated with gemcitabine, to define the correlation between the expression of hENT1 and gemcitabine efficacy in terms of PFS and OS.

\section{MATERIALS AND METHODS}

Study population. We retrospectively identified a series of 71 metastatic or locally advanced consecutive patients affected by angiosarcoma (26 cases) and leiomyosarcoma (45 cases) treated at the Istituto Nazionale Tumori of Milan, Second University of Naples, University of Palermo, Treviso General Hospital and Campus Bio-Medico University of Rome from January 2010 to December 2014. We included in the analysis only leiomyosarcoma and angiosarcoma patients with an updated follow-up and in which there was adequate naive tumour tissue to evaluate hENT1 expression.

Among the 71 patients identified, 49 patients (15 angiosarcoma and 34 leiomyosarcoma) received gemcitabine $\left(1000 \mathrm{mg} / \mathrm{m}^{2}\right.$ once weekly for 3 consecutive weeks out of every 4 weeks). Diagnosis was reviewed and confirmed by expert sarcoma pathologists (APDT, PC). The study was approved by the Biomedical Ethics Committees of the centres providing tumour tissue and an informed consent was signed by recruited patients.

A description of clinicopathologic characteristics of the entire patient cohort are summarised in Supplementary Table 1.

For all 71 patients, formalin-fixed paraffin-embedded (FFPE) surgical specimens, obtained from naive tumour at the time of diagnosis were collected. hENT1 expression levels were analysed in leiomyosarcoma and angiosarcoma patients both treated (49 patients) and not treated with gemcitabine (11 leiomyosarcoma and 11 angiosarcoma patients). All patients not treated with gemcitabine received anthracycline based regimens.

Quantitative real-time PCR. FFPE sections were treated with xylene to remove paraffin; the tissue was subsequently incubated overnight at $56^{\circ} \mathrm{C}$ with Proteinase K (Qiagen, UK) to allow sample lysis. Total RNA was extracted using the Trizol reagent (Invitrogen, CA, USA) according to the manufacturer's instructions. RNA was treated with DNAse (DNAse Turbo, Applied Biosystems, Foster City, CA, USA) to avoid genomic DNA contamination. The concentration and purity of the isolated RNA (A260/A280 ratio between 1.8 and 2.0 were accepted) were measured using a NanoDrop ND-1000 Spectrophotometer (Thermo Fisher Scientific, DE, USA). cDNA was synthetised using the high capacity cDNA Reverse Transcription Kit (Applied Biosystems) according to the manufacturer's recommendations. mRNA levels were measured by qRT-PCR performed on a 7900HT Fast Real-Time PCR System (Applied Biosystems). In all samples, hENT1 (Hs01085704_g1) expression levels were normalised to the endogenous housekeeping gene GUSb (Hs99999908_m1) using the $\Delta \mathrm{CT}$ calculation. Three technical replicates of all samples and $\mathrm{ddH}_{2} \mathrm{O}$, as non-template control, were performed and analysed for every reaction mix. Moreover, sample triplicates with $\mathrm{Ct}$ values over 35 with a $\Delta \mathrm{Ct}$ standard deviation over 0.25 were excluded to remove poor quality samples from analysis. PCR cycling included the following steps: 1 cycle at $95^{\circ} \mathrm{C}$ for $10 \mathrm{~min}, 45$ times at $95^{\circ} \mathrm{C}$ for $15 \mathrm{~s}$ and $60^{\circ} \mathrm{C}$ for $1 \mathrm{~min}$.

To confirm hENT1 protein expression, immunohistochemistry with anti-hENT1 rabbit monoclonal antibody, (SAB5500117, Sigma) was performed.

In silico analyses. To get preliminary information about hENT1 expression in leiomyosarcoma, we analysed the transcriptomic profiles of 106 patients from 'TCGA SARC' cohort from cBioportal. TCGA SARC represents the largest Sarcoma data set provided with OS information currently available even if is highly censored with a low OS event rate. All possible cutoff points have been explored using Cox proportional hazard models. In particular, survival analysis has been executed using the functions coxph and survfit from the $\mathrm{R}$ package survival. For each cutoff point, a Cox analysis of ENT1 expression level and the survival 
variable has been executed. The resulting curve of hazard ratios (HRs) is shown in Supplementary Figure 3. The percentage significant cutoffs out of all investigated cutoffs is displayed at the top of the figure. Representative cutoff point to categorise leiomyosarcoma patients into 'low' and 'high' hENT1 expression was obtained by the use of the receiver operating characteristic (ROC) method.

ROC curves are the standard method to balance between sensitivity and specificity of a molecular test. Supplementary Figure 4 shows the ROC curve for the prediction of living $\backslash$ deceased patients status by ENT1 expression level. All analysis have been performed using cutoff finder (http://molpath.charite de/cutoff). No data set for cohorts of patients affected by angiosarcoma were available from cBioportal.

Statistical analyses. For all statistical analyses the program SPSS 17.0 (SPSS, Chicago) was used. Relative changes were obtained by normalising the hENT1 mRNA expression levels to the endogenous control using the $2^{-\Delta \mathrm{Ct}}$ method (ABI software, Applied Biosystems). $2^{-\mathrm{Ct}}$ median value was used as cutoff to discriminate 'high' and 'low' hENT1 expressers.

PFS was calculated as the period from the date of starting treatment to the first observation of disease progression or to death from any cause. The OS time was calculated as the period from the date of starting treatment until death from any cause or until the date of the last follow-up, at which point data were censored. Kaplan-Meier analysis was performed to evaluate OS and PFS in patients treated with gemcitabine monotherapy by stratifying cases according to hENT1 expression level. Patients who discontinued gemcitabine for any reason without evidence of RECIST progression were censored at the last tumour assessment. Patients alive were censored at the last contact. Differences in terms of OS and PFS were evaluated according to different clinical and pathological parameters according to log-rank test. The Cox proportional hazards model was applied to the multivariate survival analysis. Differences in terms of distribution of patients features were

\begin{tabular}{|c|c|c|c|}
\hline & $\begin{array}{l}\text { High hENT1 } \\
\text { (16 patients) }\end{array}$ & $\begin{array}{l}\text { Low hENT1 } \\
\text { (18 patients) }\end{array}$ & $P$-value \\
\hline Gender (M/F) & $9 / 7$ & $11 / 7$ & 0.523 \\
\hline Median age (range) & $62(21-81)$ & $57(34-71)$ & 0.651 \\
\hline Median ECOG PS & $0(0-2)$ & $0(0-2)$ & 0.645 \\
\hline Metastatic disease & 11 & 16 & 0.214 \\
\hline $\begin{array}{l}\text { Median number of } \\
\text { metastatic sites (range) }\end{array}$ & $2(0-4)$ & $2(0-5)$ & 0.310 \\
\hline $\begin{array}{l}\text { Median number of } \\
\text { previous CTs (range) }\end{array}$ & $1(0-4)$ & $1(0-4)$ & 0.704 \\
\hline Uterine vs non-uterine & $5 / 11$ & $3 / 15$ & 0.428 \\
\hline
\end{tabular}

evaluated using fisher exact test and Mann-Whitney $U$-test when indicated. $P$-values $<0.05$ were considered statistically significant.

\section{RESULTS}

\section{Leiomyosarcoma}

hENT1 expression. hENT1 was heterogeneously expressed in leiomyosarcoma FFPE samples (median $2^{-\Delta \mathrm{Ct}}$ value 9.3 used as the cutoff to dichotomise leiomyosarcoma patients into 'high' expression and 'low' expression groups).

Table 1 summarises clinic-pathological characteristics of patients treated with gemcitabine and stratified for hENT1 expression.

Out-come analysis. Kaplan-Meier analysis in leiomyosarcoma cases treated with gemcitabine (34 patients) showed a significant correlation between hENT1 expression levels and outcome in terms of PFS (6.8 vs 3.2 months; $P=0.004)$ and OS (14.9 vs 8.5 months; $P=0.007)$ suggesting a better survival in patients with a higher hENT1 expression (Table 2; Figure 1). Conversely, in the small cohort of leiomyosarcoma patients not treated with gemcitabine (11 patients), we did not find any significant correlation between hENT1 expression and outcome (Supplementary Table 2; Supplementary Figure 1).

Moreover, the correlation between hENT1 mRNA level and protein expression was confirmed by IHC analyses. Two representative IHC images show hENT1 protein expression in two tumour samples that had high and low hENT1 mRNA levels (Figure 2).

Finally, a representative example of a hENT1 overexpressing leiomyosarcoma patient that shows major radiological response to gemcitabine treatment is depicted in Figure 3.

\section{Angiosarcoma}

hENT1 expression. hENT1 expression was less variable in angiosarcoma samples compared to leiomyosarcoma (median $2^{-\Delta \mathrm{Ct}}$ value 1.25$)$

Table 3 reports clinicopathological characteristics of gemcitabine treated patients grouped for 'high' and 'low' hENT1 expression.

Out-come analysis. Patients that exhibited high hENT1 expression showed a longer PFS (9.3 vs 4.5 months; $P=0.02)$ and a significant improvement in OS (20.6 vs 10.8 months; $P=0.001$ ) compared to those characterised by a low hENT1 expression (Table 2; Figure 4). As was the case for leiomyosarcoma, no significant association was found between hENT1 expression and PFS or OS in patients not treated with gemcitabine (Supplementary Table 2; Supplementary Figure 2).

A representative image of cutaneous localisation of hENT1 overexpressing angiosarcoma originating from the maxillary sinus before and after gemcitabine treatment is shown in Figure 5.

Table 2. Association between hENT1 expression and clinical outcome in leiomyosarcoma and angiosarcoma patients treated with gemcitabine

\begin{tabular}{|c|c|c|c|c|c|c|}
\hline & \multicolumn{2}{|c|}{$\begin{array}{l}\text { Leiomyosarcoma high hENT1 low hENT1 } \\
\text { (16 patients) (18 patients) }\end{array}$} & \multirow{2}{*}{\begin{tabular}{|c|} 
\\
$P$-value \\
0.004 \\
\end{tabular}} & \multicolumn{2}{|c|}{$\begin{array}{l}\text { Angiosarcoma high hENT1 low hENT1 (8 patients) } \\
\text { (7 patients) }\end{array}$} & \multirow{2}{*}{\begin{tabular}{|l|}
$P$-value \\
0.02
\end{tabular}} \\
\hline PFS & 6.8 months (95\% Cl 5.0-8.6) & 3.2 months (95\% Cl 2.6-3.8) & & 9.3 months $(95 \%, \mathrm{Cl} 5.9-12.9)$ & 4.5 months $(95 \% \mathrm{Cl} 3.6-5.4)$ & \\
\hline
\end{tabular}



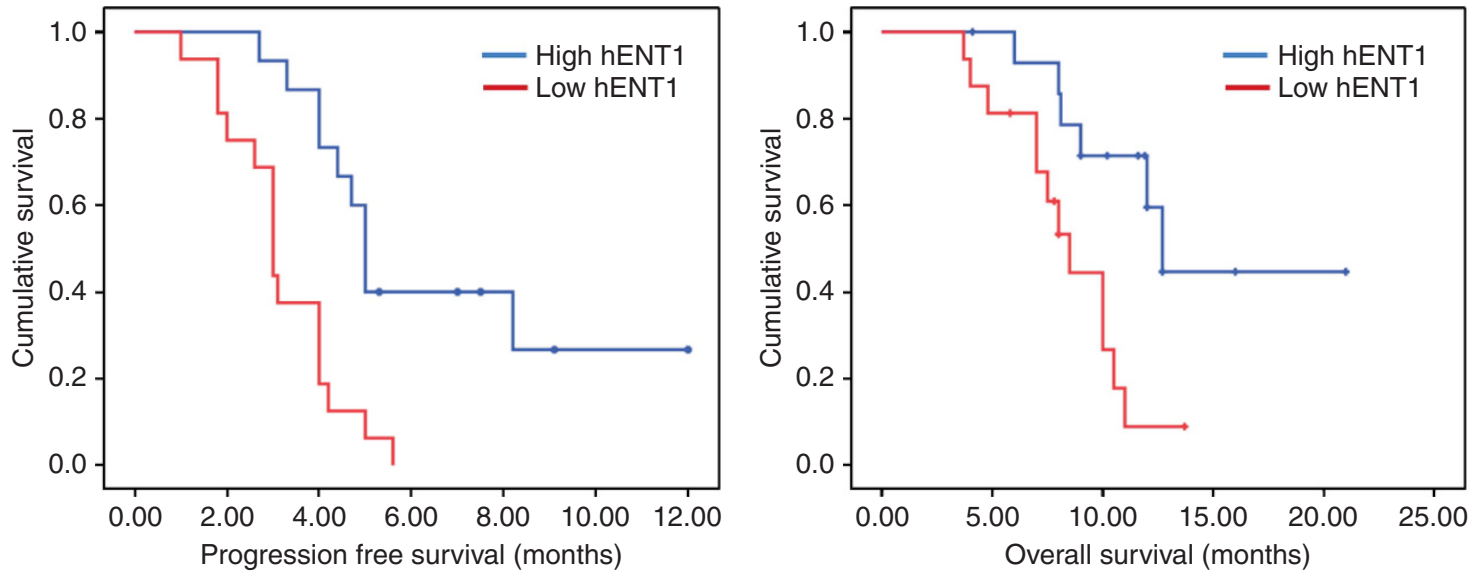

Figure 1. Kaplan-Meier curves of PFS and OS show that high hENT1 expression levels correlate with longer PFS $(P=0.004)$ and OS $(P=0.007)$ in leiomyosarcoma patients treated with gemcitabine.
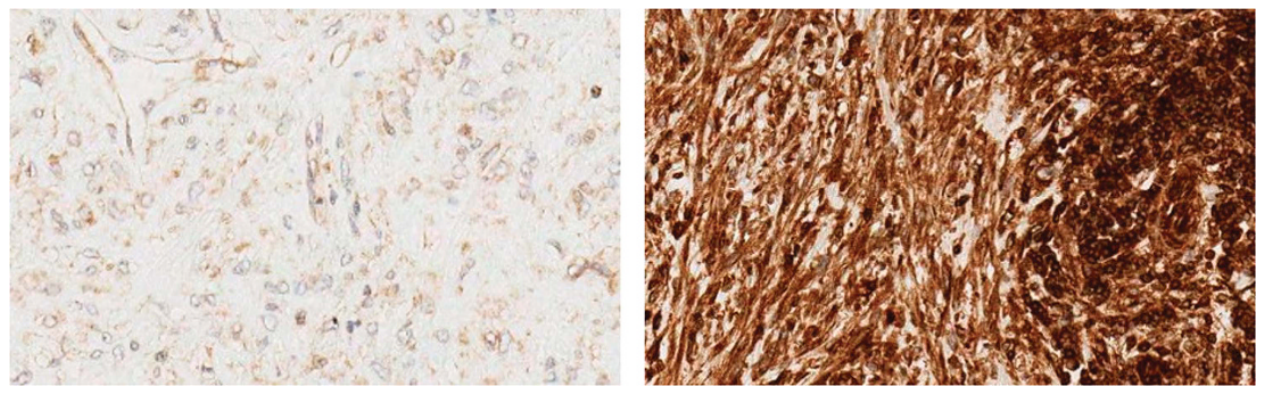

Figure 2. Representative IHC images of hENT1 protein expression in two leiomyosarcoma samples with low (left) and high (right) hENT1 mRNA level.
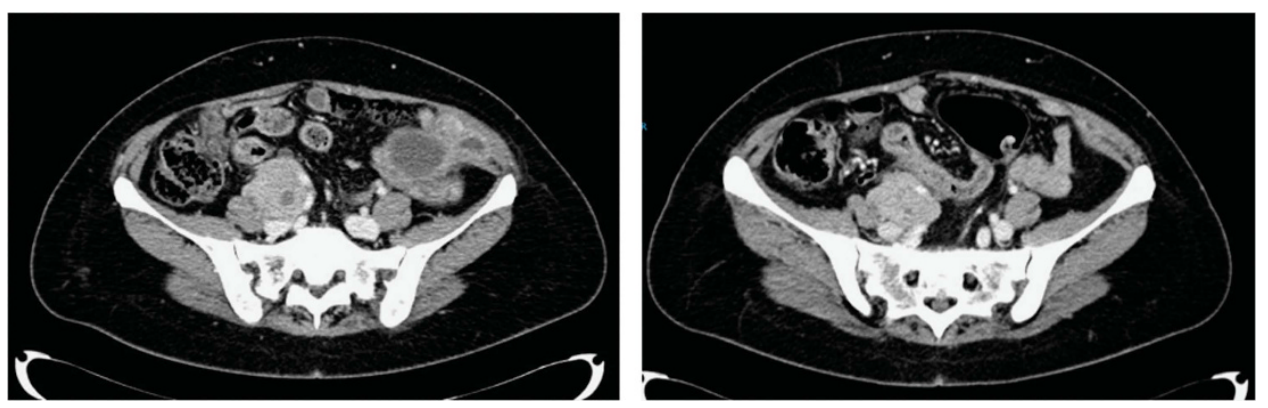

Figure 3. Baseline and 3 months CT scan in hENT1 overexpressing leiomyosarcoma patient treated with gemcitabine. This patient was diagnosed with a retroperitoneal leiomyosarcoma with multiple peritoneal nodules and lung metastasis since the first diagnosis. The patient progressed after first line anticancer therapy with adriamycin and trabectedin as second line. With gemcitabine monotherapy the patient achieved a RECIST partial response.

In silico analysis. In silico analysis showed no significant correlation between hENT1 expression levels and patients prognosis in terms of OS in a cohort of 106 patients affected by leiomyosarcoma (HR 1.21 (0.65-2.23), $P=0.55)$ (Figure 6). No data are available for angiosarcoma patients, so far.

\section{DISCUSSION}

In this retrospective study, we found that hENT1 tumour expression was associated with clinical outcome in leiomyosarcoma and angiosarcoma patients treated with gemcitabine. In particular, patients with high hENT1 expression levels showed a better outcome in terms of PFS and OS both in leiomyosarcoma (PFS: 6.8 vs 3.2 months; OS: 14.9 vs 8.5 months) and angiosarcoma (PFS was 9.3 vs 4.5 months; OS 20.6 vs 10.8 months) compared to those with low hENT1 levels. We included also 22 patients not treated with gemcitabine to evaluate and dichotomise hENT1 expression in a larger group of leiomyosarcoma and angiosarcoma patients. In this cohort of patients not treated with gemcitabine, we did not find any significant correlation between hENT1 expression and clinical outcome.

Moreover, we performed a wide in silico transcriptomic analyses in a larger cohort of leiomyosarcoma patients finding no significant association between hENT1 expression and OS. Unfortunately, no complete clinical data are available such as the number of patients who received gemcitabine. 
The association between hENT1 expression and gemcitabine efficacy was demonstrated in different tumour types including biliary tract cancer (Santini et al, 2011), cholangiocarcinoma (Borbath et al, 2012), bladder cancer (Matsumura et al, 2011) and non-small cell lung cancer (Oguri et al, 2007).

Table 3. Association between hENT1 expression and clinicopathological characteristics of angiosarcoma patients treated with gemcitabine

\begin{tabular}{|l|c|c|c|}
\hline & $\begin{array}{c}\text { High hENT1 } \\
\text { (8 patients) }\end{array}$ & $\begin{array}{c}\text { Low hENT1 } \\
\text { (7 patients) }\end{array}$ & P-value \\
\hline Gender (M/F) & $3 / 5$ & $2 / 5$ & 0.500 \\
\hline Median age (range) & $59(34-75)$ & $64(29-83)$ & 0.370 \\
\hline Median ECOG PS & $0(0-2)$ & $1(0-2)$ & 0.431 \\
\hline Metastatic disease & 6 & 5 & 0.662 \\
\hline $\begin{array}{l}\text { Median number of } \\
\text { metastatic sites (range) }\end{array}$ & $2(0-4)$ & $2(0-5)$ & 0.866 \\
\hline $\begin{array}{l}\text { Median number of } \\
\text { previous CTs (range) }\end{array}$ & 1 (0-3) & 1 (0-2) & 0.363 \\
\hline $\begin{array}{l}\text { Abbreviations: CTs = chemotherapies; ECOG PS=Performance Status according to Eastern } \\
\text { Cooperative Oncology Group; F=female; M=male. }\end{array}$ & \multicolumn{4}{l}{} \\
\hline
\end{tabular}
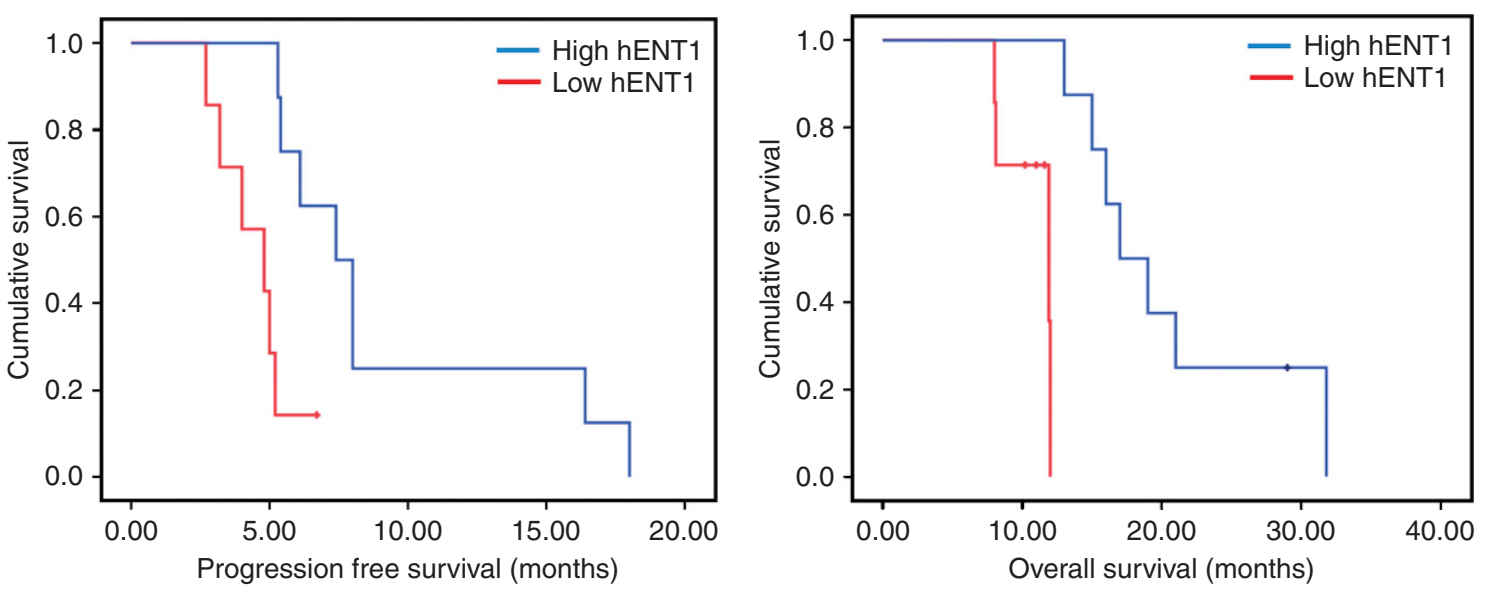

Figure 4. Kaplan-Meier curves show that high hENT1 expression levels correlate with longer PFS $(P=0.02)$ and OS $(P=0.001)$ in angiosarcoma patients treated with gemcitabine.
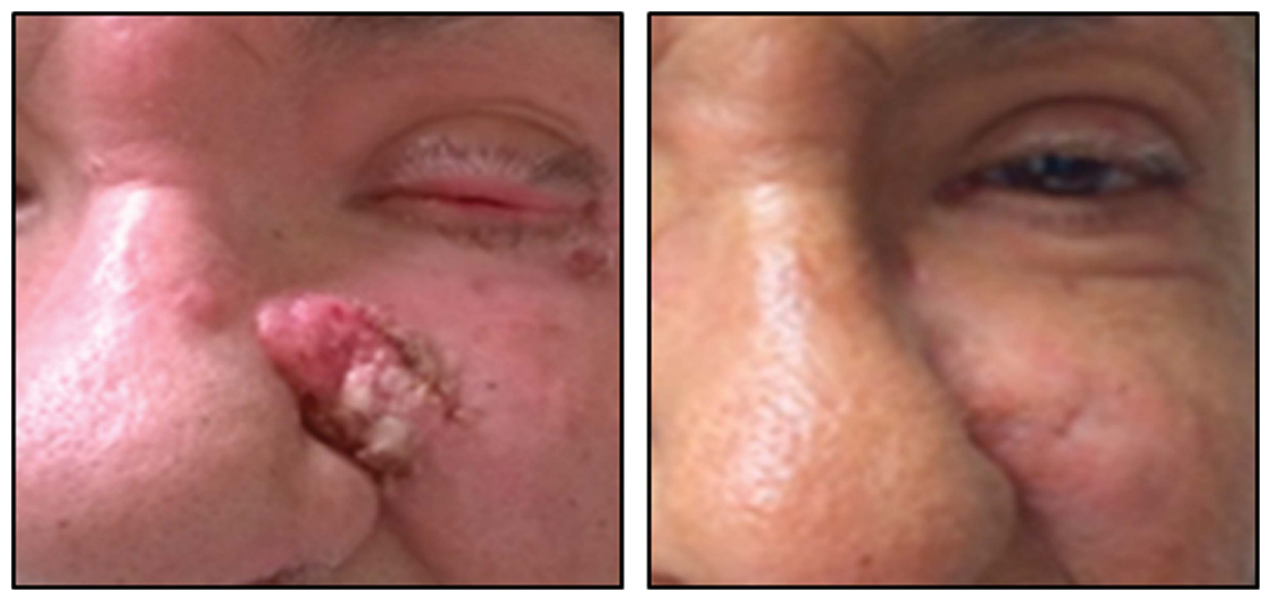

Figure 5. Tumour response in hENT1 overexpressing angiosarcoma patient treated with gemcitabine. In this patient a clinical and radiological complete response was achieved after the first three courses of gemcitabine, lasting at about 1 year. The patient is still on therapy with the same agent. 


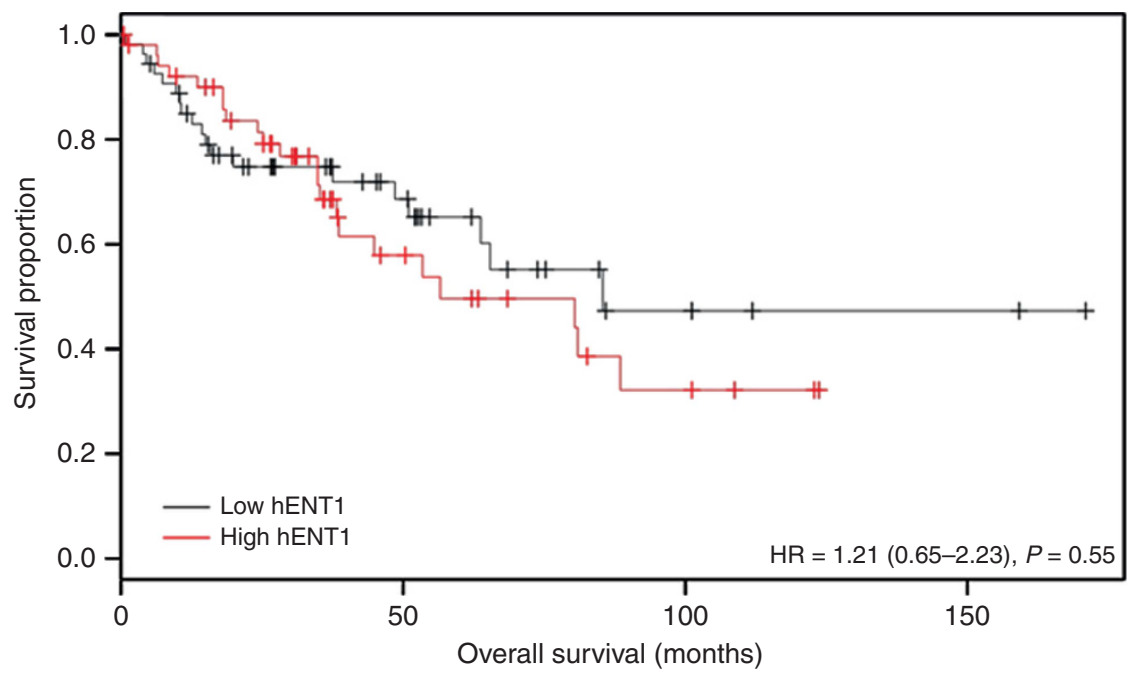

Figure 6. In silico analyses. Kaplan-Meier curves show that hENT1 expression levels did not correlate with OS in a cohort of 106 leiomyosarcoma patients.

The retrospective nature of the study represents a limitation as well as the low number of cases especially for angiosarcoma. Larger series, preferably within a prospective trial or randomised controlled trial are needed to confirm our results.

Moreover, hENT1 may not be the exclusive mediator for gemcitabine sensitivity. Indeed, other nucleoside transporter proteins are involved in the intracellular uptake of gemcitabine such as hENT3 and concentrative nucleoside transporters (Maréchal et al, 2009). Several enzymes are also implicated in the complex metabolism of gemcitabine and may contribute to the efficacy of the drug such as ribonucleotide reductase subunit 1 (RRM1, an intracellular target of gemcitabine) and deoxycytidine kinase (dCK, the enzyme that is responsible for the initial phosphorylation of gemcitabine into its active forms) (Kim et al, 2011). Thus, the combined expression analysis of hENT1 with these other markers could provide a greater predictive value than each factor alone.

In conclusion, we found that hENT1 expression could predict gemcitabine efficacy in leiomyosarcoma and angiosarcoma patients, and if confirmed in prospective trials, these data would allow a better patient selection and an improvement in therapeutic efficacy.

\section{CONFLICT OF INTEREST}

The authors declare no conflict of interest.

\section{REFERENCES}

Bhutia YD, Hung SW, Patel B, Lovin D, Govindarajan R (2011) CNT1 expression influences proliferation and chemosensitivity in drug-resistant pancreatic cancer cells. Cancer Res 71: 1825-1835.

Bjerkehagen B, Smeland S, Walberg L, Skjeldal S, Hall KS, Nesland JM, Smastuen MC, Fossa SD, Saeter G (2008) Radiation-induced sarcoma: 25-year experience from the Norwegian Radium Hospital. Acta Oncol 47: 1475-1482.

Borbath I, Verbrugghe L, Lai R, Gigot JF, Humblet Y, Piessevaux H, Sempoux C (2012) Human equilibrative nucleoside transporter 1 (hENT1) expression is a potential predictive tool for response to gemcitabine in patients with advanced cholangiocarcinoma. Eur J Cancer 48: 990-996.

Espinoza JA, García P, Bizama C, Leal JL, Riquelme I, Weber H, Macanas P, Aguayo G, Viñuela E, Roa JC, Nervi B (2016) Low expression of equilibrative nucleoside transporter 1 is associated with poor prognosis in chemotherapy-naïve pT2 gallbladder adenocarcinoma patients. Histopathology 68: 722-728.

Farrell JJ, Elsaleh H, Garcia M, Lai R, Ammar A, Regine WF, Abrams R, Benson AB, Macdonald J, Cass CE, Dicker AP, Mackey JR (2009) Human equilibrative nucleoside transporter 1 levels predict response to gemcitabine in patients with pancreatic cancer. Gastroenterology 136: 187-195.

Giovannetti E, Del Tacca M, Mey V, Funel N, Nannizzi S, Ricci S, Orlandini C, Boggi U, Campani D, Del Chiaro M, Iannopollo M, Bevilacqua G, Mosca F, Danesi R (2006) Transcription analysis of human equilibrative nucleoside transporter-1 predicts survival in pancreas cancer patients treated with gemcitabine. Cancer Res 66: 3928-3935.

Gladdy RA, Qin LX, Moraco N, Edgar MA, Antonescu CR, Alektiar KM, Brennan MF, Singer S (2010) Do radiation-associated soft tissue sarcomas have the same prognosis as sporadic soft tissue sarcomas? J Clin Oncol 28: 2064-2069.

Greenhalf W, Ghaneh P, Neoptolemos JP, Palmer DH, Cox TF, Lamb RF, Garner E, Campbell F, Mackey JR, Costello E, Moore MJ, Valle JW, McDonald AC, Carter R, Tebbutt NC, Goldstein D, Shannon J, Dervenis C, Glimelius B, Deakin M, Charnley RM, Lacaine F, Scarfe AG, Middleton MR, Anthoney A, Halloran CM, Mayerle J, Oláh A, Jackson R, Rawcliffe CL, Scarpa A, Bassi C, Büchler MW (2014) European Study Group for Pancreatic Cancer. Pancreatic cancer hENT1 expression and survival from gemcitabine in patients from the ESPAC-3 trial. J Natl Cancer Inst 106: djt347.

Hensley ML, Blessing JA, Mannel R, Rose PG (2008) Fixed-dose rate gemcitabine plus docetaxel as first-line therapy for metastatic uterine leiomyosarcoma: a Gynecologic Oncology Group phase II trial. Gynecol Oncol 109: 329-334.

Hensley ML, Maki R, Venkatraman E, Geller G, Lovegren M, Aghajanian C, Sabbatini P, Tong W, Barakat R, Spriggs DR (2002) Gemcitabine and docetaxel in patients with unresectable leiomyosarcoma: results of a phase II trial. J Clin Oncol 20: 2824-2831.

Kawada N, Uehara H, Katayama K, Nakamura S, Takahashi H, Ohigashi H, Ishikawa O, Nagata S, Tomita Y (2012) Human equilibrative nucleoside transporter 1 level does not predict prognosis in pancreatic cancer patients treated with neoadjuvant chemoradiation including gemcitabine. $J$ Hepatobiliary Pancreat Sci 19: 717-722. Erratum in: J Hepatobiliary Pancreat Sci 19: 723.

Kim R, Tan A, Lai KK, Jiang J, Wang Y, Rybicki LA, Liu X (2011) Prognostic roles of human equilibrative transporter 1 (hENT-1) and ribonucleoside reductase subunit M1 (RRM1) in resected pancreatic cancer. Cancer 117: 3126-3134.

Maréchal R, Bachet JB, Mackey JR, Dalban C, Demetter P, Graham K, Couvelard A, Svrcek M, Bardier-Dupas A, Hammel P, Sauvanet A, Louvet C, Paye F, Rougier P, Penna C, André T, Dumontet C, Cass CE, Jordheim LP, Matera EL, Closset J, Salmon I, Devière J, Emile JF, Van Laethem JL (2012) Levels of gemcitabine transport and metabolism proteins predict survival times of patients treated with gemcitabine for pancreatic adenocarcinoma. Gastroenterology 143: 664-674e1-6. 
Maréchal R, Mackey JR, Lai R, Demetter P, Peeters M, Polus M, Cass CE, Young J, Salmon I, Devière J, Van Laethem JL (2009) Human equilibrative nucleoside transporter 1 and human concentrative nucleoside transporter 3 predict survival after adjuvant gemcitabine therapy in resected pancreatic adenocarcinoma. Clin Cancer Res 15: 2913-2919.

Matsumura N, Nakamura Y, Kohjimoto Y, Inagaki T, Nanpo Y, Yasuoka H, Ohashi Y, Hara I (2011) The prognostic significance of human equilibrative nucleoside transporter 1 expression in patients with metastatic bladder cancer treated with gemcitabine-cisplatin-based combination chemotherapy. BJU Int 108: E110-E116.

Michalski CW, Erkan M, Sauliunaite D, Giese T, Stratmann R, Sartori C, Giese NA, Friess H, Kleeff J (2008) Ex vivo chemosensitivity testing and gene expression profiling predict response towards adjuvant gemcitabine treatment in pancreatic cancer. Br J Cancer 99: 760-767.

Morinaga S, Nakamura Y, Watanabe T, Mikayama H, Tamagawa H, Yamamoto N, Shiozawa M, Akaike M, Ohkawa S, Kameda Y, Miyagi Y (2012) Immunohistochemical analysis of human equilibrative nucleoside transporter-1 (hENT1) predicts survival in resected pancreatic cancer patients treated with adjuvant gemcitabine monotherapy. Ann Surg Oncol 19: S558-S564.

Nakagawa N, Murakami Y, Uemura K, Sudo T, Hashimoto Y, Kondo N, Sueda T (2013) Combined analysis of intratumoral human equilibrative nucleoside transporter 1 (hENT1) and ribonucleotide reductase regulatory subunit M1 (RRM1) expression is a powerful predictor of survival in patients with pancreatic carcinoma treated with adjuvant gemcitabinebased chemotherapy after operative resection. Surgery 153: 565-575.

Oguri T, Achiwa H, Muramatsu H, Ozasa H, Sato S, Shimizu S, Yamazaki H, Eimoto T, Ueda R (2007) The absence of human equilibrative nucleoside transporter 1 expression predicts nonresponse to gemcitabine-containing chemotherapy in non-small cell lung cancer. Cancer Lett 256: 112-119.

Pautier P, Floquet A, Penel N, Piperno-Neumann S, Isambert N, Rey A, Bompas E, Cioffi A, Delcambre C, Cupissol D, Collin F, Blay JY, Jimenez M, Duffaud F (2012) Randomized multicenter and stratified phase II study of gemcitabine alone versus gemcitabine and docetaxel in patients with metastatic or relapsed leiomyosarcomas: a Federation Nationale des Centres de Lutte Contre le Cancer (FNCLCC) French Sarcoma Group Study (TAXOGEM study). Oncologist 17: 1213-1220.

Pérez-Torras S, García-Manteiga J, Mercadé E, Casado FJ, Carbó N, Pastor-Anglada M, Mazo A (2008) Adenoviral-mediated overexpression of human equilibrative nucleoside transporter 1 (hENT1) enhances gemcitabine response in human pancreatic cancer. Biochem Pharmacol 76: 322-329.

Perrone G, Morini S, Santini D, Rabitti C, Vincenzi B, Alloni R, Antinori A, Magistrelli P, Lai R, Cass C, Mackey JR, Coppola R, Tonini G, Onetti Muda A (2010) Human equilibrative nucleoside transporter 1 and carcinoma of the ampulla of Vater: expression differences in tumour histotypes. Eur J Histochem 54: e38.

Poplin E, Wasan H, Rolfe L, Raponi M, Ikdahl T, Bondarenko I, Davidenko I, Bondar V, Garin A, Boeck S, Ormanns S, Heinemann V, Bassi C, Evans TR, Andersson R, Hahn H, Picozzi V, Dicker A, Mann E, Voong C, Kaur P, Isaacson J, Allen A (2013) Randomized, multicenter, phase II study of CO-101 versus gemcitabine in patients with metastatic pancreatic ductal adenocarcinoma: including a prospective evaluation of the role of hENT1 in gemcitabine or CO-101 sensitivity. J Clin Oncol 31: 4453-4461.

Santini D, Perrone G, Vincenzi B, Lai R, Cass C, Alloni R, Rabitti C, Antinori A, Vecchio F, Morini S, Magistrelli P, Coppola R, Mackey JR, Tonini G (2008) Human equilibrative nucleoside transporter 1 (hENT1) protein is associated with short survival in resected ampullary cancer. Ann Oncol 19: 724-728.

Santini D, Schiavon G, Vincenzi B, Cass CE, Vasile E, Manazza AD, Catalano V, Baldi GG, Lai R, Rizzo S, Giacobino A, Chiusa L, Caraglia M, Russo A, Mackey J, Falcone A, Tonini G (2011) Human equilibrative nucleoside transporter 1 (hENT1) levels predict response to gemcitabine in patients with biliary tract cancer (BTC). Curr Cancer Drug Targets 11: 123-129.

Santini D, Vincenzi B, Fratto ME, Perrone G, Lai R, Catalano V, Cass C, Ruffini PA, Spoto C, Muretto P, Rizzo S, Muda AO, Mackey JR, Russo A, Tonini G, Graziano F (2010) Prognostic role of human equilibrative transporter 1 (hENT1) in patients with resected gastric cancer. J Cell Physiol 223: 384-388.

Spratlin J, Sangha R, Glubrecht D, Dabbagh L, Young JD, Dumontet C, Cass C, Lai R, Mackey JR (2004) The absence of human equilibrative nucleoside transporter 1 is associated with reduced survival in patients with gemcitabine-treated pancreas adenocarcinoma. Clin Cancer Res 10: 6956-6961.

Stacchiotti S, Palassini E, Sanfilippo R, Vincenzi B, Arena MG, Bochicchio AM, De Rosa P, Nuzzo A, Turano S, Morosi C, Dei Tos AP, Pilotti S, Casali PG (2012) Gemcitabine in advanced angiosarcoma: a retrospectivecase series analysis from the Italian Rare CancerNetwork. Ann Oncol 23: 501-508.

Ueno H, Kiyosawa K, Kaniwa N (2007) Pharmacogenomics of gemcitabine: can genetic studies lead to tailor-made therapy? Br J Cancer 97: 145-151.

This work is published under the standard license to publish agreement. After 12 months the work will become freely available and the license terms will switch to a Creative Commons AttributionNonCommercial-Share Alike 4.0 Unported License.

Supplementary Information accompanies this paper on British Journal of Cancer website (http://www.nature.com/bjc) 